\title{
Alta prevalencia de hernias abdominales en pacientes con síndrome velocardiofacial
}

\author{
JOSÉ ANDRÉS JUSTINIANO D. ${ }^{1}$, MARÍA LUISA GUZMÁN G. ${ }^{2}$, \\ CARMEN PAZ ASTETE A. ${ }^{3}$, TERESA ARAVENA C. ${ }^{4,5}$, MARTA ARRIAZA Z.$^{6}$, \\ MARIANA ARACENA A., ${ }^{3,7}$, M. GABRIELA REPETTO L. ${ }^{8,9,10}$ \\ 1. Estudiante de Medicina, Facultad de Medicina, Clínica Alemana-Universidad del Desarrollo. \\ 2. Enfermera, Centro de Genética Humana, Facultad de Medicina, Clínica Alemana-Universidad del Desarrollo. \\ 3. Genetista Clínico, Hospital Luis Calvo Mackenna. \\ 4. Genetista Clínico, Complejo Hospitalario Dr. Sótero del Río. \\ 5. Genetista Clínico, INTA, Universidad de Chile. \\ 6. Genetista Clínico, Hospital Gustavo Fricke. \\ 7. División de Pediatría, Pontificia Universidad Católica de Chile. \\ 8. Directora, Centro de Genética Humana, Facultad de Medicina, Clínica Alemana-Universidad del Desarrollo. \\ 9. Genetista Clínico, Unidad de Gestión Clínica del Niño, Hospital Padre Hurtado. \\ 10.Genetista Clínico, Depto de Pediatría, Clínica Alemana.
}

\begin{abstract}
High incidence of abdominal wall hernias in patients with velocardiofacial syndrome
\end{abstract}

Velocardiofacial syndrome (VCFS) is due to a microdeletion on chromosome region 22q11.2. Clinically, it is characterized by congenital anomalies and psychiatric and cognitive manifestations. The most common structural defects are congenital heart disease and palatal anomalies, both due to abnormal development of the pharyngeal pouches. Another less studied manifestation is abdominal wall hernias. Objective: To characterize the frequency and types of hernias in patients with VCFS, and their association with congenital cardiopathies and palatine abnormalities. Patients and Methods: 202 patients were evaluated by direct clinical examination and questionnaire about their phenotypic characteristics. Results were compared to those found in the literature. Results: Age range was 0.5 to 48.4 years old (mean 11.9 years), 50.4\% were females. Twenty two percent of patients had abdominal wall hernias. Of these, $49.1 \%$ were inguinal and $40.3 \%$, umbilical. Conclusion: Patients with VCFS have a higher incidence of abdominal hernias than general population, described as approximately $5 \%$. This is another common manifestation of the syndrome, not attributable to defects in development of pharyngeal pouches and with unknown pathogenesis.

(Key words: Abdominal wall, congenital heart disease, hernia, velocardiofacial syndrome, 22q11 microdeletion).

Rev Chil Pediatr 2012; 83 (1): 42-47

Trabajo recibido el 30 de julio de 2011, devuelto para corregir el 28 de agosto de 2011, segunda versión el 5 de octubre de 2011, aceptado para publicación el 30 de enero de 2012.

Financiado por Fondecyt, proyectos \# 1061051 y 1100131.

Correspondencia a:

Dra. Gabriela Repetto L.

E-mail: grepetto@udd.cl 


\section{RESUMEN}

El síndrome velocardiofacial (SVCF) se debe a una microdeleción en la región cromosómica 22q11.2. Clínicamente, se caracteriza por anomalías congénitas y manifestaciones siquiátricas y cognitivas. Entre las malformaciones más comunes, están las cardiopatías congénitas y anomalías palatinas, por defectos en el desarrollo de las bolsas faríngeas. Otra manifestación menos estudiada son las hernias de la pared abdominal. Objetivo: Caracterizar la frecuencia y tipos de hernias en pacientes con SVCF y su asociación con cardiopatías congénitas y anomalías del paladar. Pacientes y Método: Evaluamos 202 pacientes mediante un examen clínico directo y un cuestionario sobre sus características fenotípicas. Comparamos los resultados con la información de la literatura. Resultados: El rango de edad fue de 0,5 a 48,4 años (media de 11,9 años), 50,4\% de sexo femenino. El 22\% de los pacientes presentó hernias de la pared abdominal. De estas, el 49,1\% fueron inguinales y el 40,3\%, umbilicales. La frecuencia de hernias en los pacientes con SVCF es significativamente mayor que la descrita para la población general, aproximadamente un 5\%. Esta es una manifestación común del síndrome, que no es atribuible a defectos del desarrollo de las bolsas faríngeas y cuya patogenia no ha sido definida.

(Palabras clave: Pared abdominal, cardiopatía congénita, hernia, síndrome velocardiofacial, microdeleción 22q11).

Rev Chil Pediatr 2012; 83 (1): 42-47

\section{Introducción}

El síndrome velocardiofacial (SVCF) es una de las alteraciones genéticas más comunes en los humanos. Según los datos de la VeloCardio-Facial Syndrome Educational Foundation se estima que este síndrome se manifiesta en 1 de cada 2 000-4 000 personas $^{1}$. Se debe a una microdeleción en la región cromosómica 22q11.2 2 .3.

Clínicamente, el SVCF se caracteriza por anomalías congénitas y manifestaciones siquiátricas y cognitivas ${ }^{1,2}$. Entre las malformaciones más comunes, están las cardiopatías congénitas y anomalías palatinas, las que se originan por defectos en el desarrollo del aparato faríngeo embrionario ${ }^{2,4,5}$. Se han descrito centenares de otras manifestaciones en prácticamente todos los órganos y sistemas ${ }^{1}$, que incluyen infecciones recurrentes por inmunodeficiencia, dificultades en el aprendizaje, dificultad en la alimentación e hipocalcemia, entre otras $^{6}$. El diagnóstico del síndrome se basa en la sospecha clínica y la confirmación se realiza mediante técnica de hibridación in situ con fluorescencia (FISH) con sondas específicas para la región cromosómica 22q11.2.

Entre las manifestaciones que han sido menos estudiadas están las hernias de la pared abdominal ${ }^{1,6}$, las cuales son el objeto de esta investigación. Una hernia de la pared abdominal es la protrusión de peritoneo parietal (que puede acompañarse de vísceras intra-abdominales) a través de un orificio o anillo anatómicamente débil de la pared abdominal ${ }^{7}$.

Nuestra hipótesis es que los pacientes con SVCF tienen una mayor frecuencia de hernias de la pared abdominal que el resto de la población. El objetivo fue caracterizar la frecuencia y tipos de hernias de la pared abdominal en pacientes con SVCF, y su asociación con cardiopatías congénitas y anomalías del paladar.

\section{Pacientes y Método}

Se estudiaron pacientes con SVCF confirmado mediante técnica de FISH. Participaron pacientes de cuatro hospitales terciarios de Santiago y uno de Viña del Mar, Chile. Estos centros atienden a pacientes con SVCF derivados de diferentes regiones del país. La información se obtuvo mediante examen físico directo, revisión de fichas clínicas y cuestionarios estandarizados, realizados a los pacientes, padres y/o sus médicos tratantes, que describen las características clínicas de los pacientes con del22q11.2. En ausencia de un registro nacional que indique datos precisos de las hernias, comparamos los resultados con la información 
de la literatura interpolada para la población general en Chile ${ }^{7}$. La información recopilada se ingresó en una base de datos y se analizó con Microsoft Excel 2007.

Se recolectó información de la anatomía cardíaca por ultrasonido, cateterismo cardíaco y/o resonancia magnética; la estructura del paladar y su función por otorrinolaringólogo, cirujano plástico y/o fonoaudiólogo, evaluación por nasofaringoscopía cuando estuvo indicada y de las hernias por examen físico de la pared abdominal. Los resultados se compararon con la frecuencia estimada de hernias de pared abdominal para la población chilena ${ }^{8}$, basada en las prevalencias de hernias de la Guía Clínica de hernias de la pared abdominal del Ministerio de Salud ${ }^{7}$. Un valor p de 0,05 fue considerado como una diferencia estadísticamente significativa. El estudio fue aprobado por el Comité de Ética de las instituciones participantes, y los pacientes o sus padres dieron por escrito su consentimiento informado.

\section{Resultados}

La población total estudiada fue de 202 pacientes con SVCF confirmado por FISH, con información concerniente a la presencia o ausencia de hernias. La edad de diagnóstico clínico promedio del síndrome fue de 5,6 años y la edad actual promedio de los pacientes al momento de realizar el estudio fue de 11,9 años, con una mediana de 10,25 años. Las características demográficas y clínicas se resumen en la tabla 1. Otras manifestaciones de este grupo de pacientes han sido previamente publicadas ${ }^{9}$.

Contamos con datos sobre presencia o ausencia de hernias en los 202. Cuarenta y cuatro pacientes (22\%) tuvieron hernias abdominales, lo que corresponde a un odds ratio (OR) de 5,3 (IC 95\% de 3,8-7,4) para SVCF en comparación al estimado de 5\% para la población general chilena ${ }^{8}$. En cuanto al tipo de hernias, la más frecuente fue la inguinal, vista en 28 pacientes o $49 \%$ de los pacientes con hernias. El resto de los pacientes tuvo hernias umbilicales e inguinales (7 pacientes, correspondientes al $12,3 \%$ del total de las hernias y 3,5\% del total de pacientes), supraumbilicales o de la línea media o epigástricas (4 pacientes, correspondientes al $7 \%$ y $2 \%$ respectivamente) o hernias de ubicación no registrada (2 pacientes, correspondientes al 3,5\% y $1 \%$, respectivamente). De las hernias inguinales, $38,1 \%$ fueron izquierdas, 42,9\% derechas, 28,6\% de ambas y 19,04\% de localización no registrada.

Las hernias umbilicales fueron más frecuentes en los pacientes con SVCF que la cifra descrita en las normas de Garantías Explicitas en Salud (GES)), resultando en un OR de 6 (IC 95\%: 3,6-10,3). En el caso de las hernias inguinales fueron menos frecuentes en los pacientes con SVCF, resultando en un OR de 0,24 (IC 95\%: 0,14-0,4).

En relación a las anomalías congénitas más comunes del síndrome, contaron con datos sobre anatomía cardíaca 201 pacientes, del paladar 159 y, de ambas anomalías congénitas, 158. El 57\% tuvo cardiopatía congénita; 70,4\%, anomalía del paladar; y 33,5\% tuvo ambas. Analizamos la asociación de hernias con estas anomalías. La hernia más frecuentemente asociada a la presencia de anomalía del paladar o cardiopatía congénita fue la umbilical (55\% y $39 \%$, respectivamente). A la inversa, la alteración congénita más frecuente en los pacientes con y sin hernia fue la anomalía del paladar (tabla 2). Como se puede observar en la figura 1 , la cardiopatía congénita más frecuente en pacientes con hernia fue la comunicación interventricular. La frecuencia de los distintos tipos de anomalías del paladar fue similar para pacientes con y sin hernias (figura 2).

Tabla 1. Caracterización de los pacientes con SVCF incluidos en este estudio ( $n$ : 202)

\begin{tabular}{|lll|}
\hline Edad (años) & Rango & $0,5-48,4$ \\
& Media & 11,9 \\
& Mediana & 10,25 \\
\hline Sexo, n (\%) & Mujer & $102(50,4)$ \\
& Hombre & $100(49,6)$ \\
Fallecidos, n (\%) & & $10(5)$ \\
\hline Hernias, $\mathrm{n}(\%)$ & & $44(22)$ \\
\hline
\end{tabular}



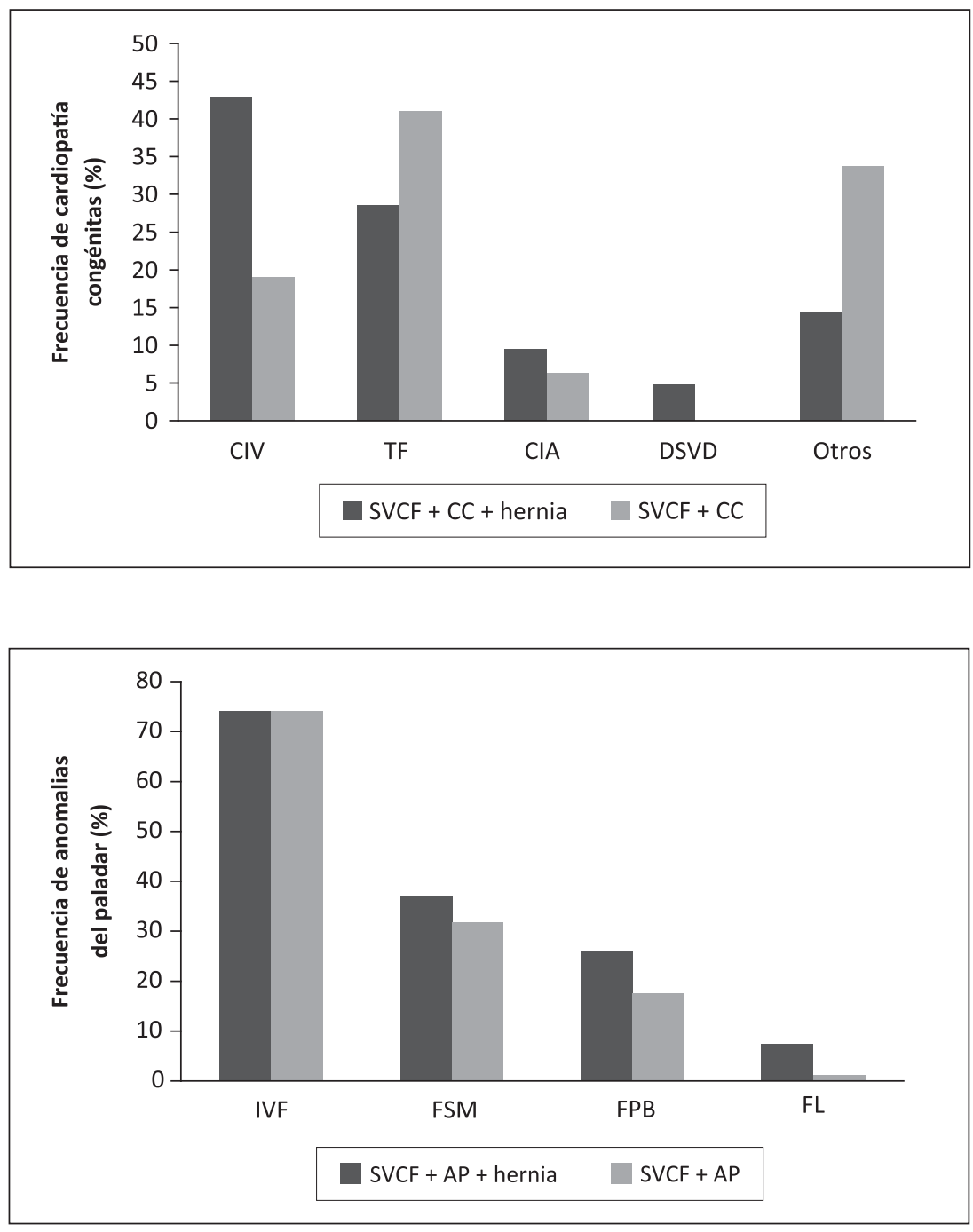

Figura 1. Tipos de cardiopatías congénitas en pacientes con SVCF, con y sin hernia (n: 21 y n: 95, respectivamente). Diferencia estadísticamente significativa para CIV (OR: 3,2; IC 95\%:1,8-8,8; p < 0,05). CC: Cardiopatía Congénita; CIV: Comunicación Interventricular; TF: Tetralogía de Fallot; CIA: Comunicación Interauricular; DSVD: Doble Salida Ventricular Derecha.
Tabla 2. Anomalías cardíacas y palatinas en pacientes con (33) y sin hernias (125)

\begin{tabular}{|lccc|}
\hline & $\begin{array}{c}\text { Cardiopatía } \\
\text { congénita }\end{array}$ & $\begin{array}{c}\text { Anomalía } \\
\text { del paladar }\end{array}$ & Ambas \\
\hline $\begin{array}{l}\text { Pacientes con } \\
\text { hernias, } n(\%)\end{array}$ & $13(39)$ & $27(82)$ & $10(30)$ \\
\hline $\begin{array}{l}\text { Pacientes sin } \\
\text { hernias, } \mathrm{n}(\%)\end{array}$ & $66(53)$ & $85(68)$ & $38(30)$ \\
\hline
\end{tabular}

\section{Discusión}

En este trabajo se evaluó a un gran número de pacientes con SVCF, comparable a otras series internacionales ${ }^{10-13}$. La frecuencia de las principales malformaciones congénitas, cardiopatías y anomalías del paladar, fue similar a la frecuencia en otros estudios extranjeros ${ }^{6,14}$.

Observamos que hubo una alta incidencia de hernias de la pared abdominal en la población estudiada, más de 5 veces mayor que la frecuencia de la población general. Esto evidencia que los pacientes con SVCF tienen más predisposición para formar hernias que la población general. Si bien la presencia de esta malformación en los pacientes con del 22q11.2 ha sido mencionada en la literatura sobre el síndrome ${ }^{15}$, este es el primer estudio que cuantifica la frecuencia de hernias en un grupo grande de pacientes con SVCF. 
La frecuencia de los principales tipos de hernias (umbilical e inguinal) en pacientes con SVCF fue distinta a la publicada para la población general. Si bien en ambos grupos las hernias inguinales son más frecuentes que las umbilicales, en los pacientes con SVCF encontramos una proporción mayor de hernias umbilicales en comparación con la población general.

Se han descrito algunos factores predisponentes para la formación de hernias entre los que se encuentran factores metabólicos (como la disminución en la formación o formación deficiente de colágeno y el aumento de la degradación de colágeno), anatómicos (como la ausencia de las fibras aponeuróticas del músculo transverso abdominal) y fisiológicos o mecánicos (como deficiente acción del diafragma muscular o aumentos de la presión intra-abdominal por obesidad) ${ }^{16}$. En el caso de los pacientes con microdeleción de la región cromosómica 22q11.2, la causa de las hernias no ha sido identificada. Pueden deberse al déficit del gen TBX1, que codifica para el factor de transcripción T-box1, al cual se ha atribuido la mayoría de las anomalías estructurales del síndrome debido por su rol en la formación de la cara y tracto de salida cardíaco. Existe evidencia de que este gen participa en el desarrollo de la musculatura esquelética ${ }^{17}$ pudiendo afectar la resistencia de la pared abdominal. Por otra parte, hay otros genes deletados en este síndrome que podrían explicar la predisposición a hernias, como el gen CRKL que codifica para un protooncogen CRK-símil, el cual es relevante para el desarrollo del sistema nervioso central $^{18}$. Participa en vías de transducción y transformación celular ${ }^{19}$ y está involucrado en los mecanismos de adhesión celular a la matriz extracelular $^{20-22}$, lo que lo hacen un potencial candidato para esta manifestación del síndrome. Alternativamente, podría haber otras alteraciones genéticas que afecten el desarrollo de la pared abdominal alterando la síntesis de colágeno, elastina u otros factores de la matriz extracelular.

Este estudio tiene algunas limitaciones: evaluó a pacientes en los que se ha reconocido y confirmado el diagnóstico de SVCF, que pueden no necesariamente ser representativos del universo de pacientes con la deleción, y comparó los hallazgos con una estimación de la frecuencia de hernias abdominales en la población chilena, basada en los datos que utiliza el Ministerio de Salud a partir de cifras de otros países ${ }^{7}$ ya que no existen registros que aporten datos precisos de éstas (comunicación personal de la Jefa del Departamento de Estadísticas e Información de Salud del Ministerio de Salud de Chile, Dra. Danuta Rajs, consultada en septiembre del año 2010). Estas cifras además no distinguen pacientes pediátricos y adultos. Sin embargo, este trabajo tiene como ventajas el gran número de pacientes evaluados, y que se realizó un seguimiento activo, obteniendo información de múltiples fuentes (ficha clínica, examen directo, cuestionario a padres y médicos tratantes).

Sugerimos que la búsqueda dirigida de hernias de la pared abdominal debe ser parte del seguimiento clínico de los pacientes con SVCF. Proponemos que, el hallazgo de hernia y cardiopatía congénita o de hernia y fisura palatina en un paciente, debería hacer considerar el diagnóstico de SVCF.

\section{Agradecimientos}

A Juan Eduardo Justiniano D., Ingeniero Civil, por su colaboración en el desarrollo del análisis estadístico de datos.

\section{Referencias}

1.- $\quad$ The Velo-Cardio-Facial Syndrome Educational Foundation [Internet]: Velo-cardio-facial syndrome, Specialist fact sheet. Citado el 16-01-11. Disponible en: http:// www.vcfsef.org.

2.- Matsuoka R, Kimura M, Scambler PJ, et al: Molecular and clinical study of 183 patients with conotruncal anomaly face syndrome. Human Genet; 1998; 103: 70-80.

3.- Klewer SE, Runyan RB, Erickson RP: TBX1 and the DiGeorge Syndrome Critical Region. Epstein C.J., Erickson R. P., Wynshaw-Boris A. Ed. Inborn errors of development: the molecular basis of clinical disorders of morphogenesis, Oxford: Oxford University Press US, 2004; 699-704.

4.- Vermot J, Niederreither K, Garnier J, Chambon P, Dolle $P$ : Decreased embryonic retinoic acid synthesis results 
in a Di George syndrome phenotype in newborn mice. Proc Natl Acad Sci USA; 2002; 100: 1763-8.

5.- Goodman FR: Congenital abnormalities of body patterning: embryology revisited. Lancet 2003; 362: 351-62.

6.- Óskarsdóttir S, Persson C, Eriksson BO, Fasth A: Presenting phenotype in 100 children with the 22q11 deletion syndrome. Eur J Pediatr; 2005; 164: 146-53.

7.- Garantías Explícitas en Salud. Guías Clínicas Problemas AUGE. MINSAL: Guía clínica hernias de la pared abdominal, 2008, citado el 16-01-11. Disponible en: http://www.redsalud.gov.cl

8.- Instituto Nacional de Estadística. Estadísticas Demográficas y vitales: Chile: Proyecciones y Estimaciones de Población. Total País. 1950-2050, 2008, citado el 16-01-11. Disponible en: http://www.ine.cl

9.- Repetto GM, Guzmán ML, Puga A, et al: Clinical features of chromosome 22q11.2 microdeletion syndrome in 208 Chilean patients. Clin Genet 2009; 76: 465-70.

10.- Machado RF, Gazzola PR, Pires C, et al: 22q11.2 Duplication and Congenital Heart Defects. Arq Bras Cardiol; 2009; 93: e55-7.

11.- Goldmuntz E, Driscoll DA, Emanuel BS, et al: Evaluation of Potential Modifiers of the Cardiac Phenotype in the 22q11.2 Deletion Syndrome. Birth Defects Res A Clin Mol Teratol 2009; 85: 125-9.

12.- McElhinney DB, Driscoll DA, Levin ER, Jawad AF, Emanuel BS, Goldmuntz E: Chromosome 22q11 Deletion in Patients With Ventricular Septal Defect: Frequency and Associated Cardiovascular Anomalies. Pediatrics 2003; 112: e472-6.

13.- Kwiatkowska J, Wierzba J, Aleszewicz-Baranowska J, Erecinki J: Genetic background of congenital conotruncal heart defects-a study of 45 families. Kardiol Pol;
2007; 65: 32-7.

14.- Ryan AK, Goodship JA, Wilson DI, et al: Spectrum of clinical features associated with interstitial chromosome 22ql 1 deletions: a European collaborative study. J Med Genet 1997; 34: 798-804.

15.- Bassett AS, McDonald-McGinn DM, Devriendt K, et al: Practical Guidelines for Managing Patients with 22q11.2 Deletion Syndrome. J Pediatr 2011; 159: 3329.

16.- Asociación Mexicana de Hernia: Guías clínicas de hernia de pared de la AMH: Guía de práctica clínica para hernias de la pared abdominal, 2009, citado el 16-01-11. Disponible en: http:// www.amhernia.org/

17.- Online Mendelian Inheritance in Man, OMIM [Internet]: T-BOX 1; TBX1. Citado el 15-06-11. Disponible en: www.ncbi.nlm.nih.gov

18.- Park T, Curran T: Crk and CrkL play essential overlapping roles downstream of Dab1 in the Reelin pathway. J Neurosci 2008; 28: 13551-62.

19.- Senechal K, Halpern J, Sawyers CL: The CRKL Adaptor Protein Transforms Fibroblasts and Functions in Transformation by the BCR-ABL Oncogene. J Biol Chem 1996; 271: 23255-61.

20.- Uemura N, Salgia R, Ewaniuk DS, Little MT, Griffin JD: Involvement of the adapter protein CRKL in integrinmediated adhesion. Oncogene; 1999; 18: 3343-53.

21.- Moon AM, Guris $\mathrm{DL}$, Seo $\mathrm{J}-\mathrm{H}$, et al: Crkl deficiency disrupts Fgf8 signaling in a mouse model of 22q11 deletion syndrome. Dev Cell 2006; 10: 71-80.

22.- Guris DL, Duester G, Papaioannou VE, Imamoto A: Dose-dependent interaction of Tbx1 and Crkl and locally aberrant RA signaling in a model of del22q11 syndrome. Dev Cell 2006; 10: 81-92. 